\title{
Myoepithelial carcinoma of mixed cell type: a rare entity
}

Keywords: merkel-cell carcinoma, calponin, CD99, myoepithelial carcinomas, GFAP

\section{Introduction}

Myoepitheliomas represent a large group of uncommon mesenchymal neoplasms characterized by myoepithelial differentiation. ${ }^{1}$

Soft tissue myoepitheliomas are histologically characterized by a wide spectrum of morphological and immunophenotypic features; the majority consists of different cell populations (i.e. epithelioid, spindled or clear cells), variably expressing epithelial markers (EMA/ cytokeratin), S100 and GFAP. Spindled and epithelioid cells can be documented in different areas of the same tumor (biphasic pattern) (Figure 1), and cells with intermediate features are frequently present in the intervening zones. Neoplastic cells are organized in a solid, nested or reticular growth pattern and foci of collagenous or mixoid stroma is commonly observed. ${ }^{2,3}$ The presence of necrosis and/or a high mitotic activity are clues for the diagnosis of malignant myoepitheliomas. ${ }^{4}$ In some myoepithelial carcinomas (MECa), the cells focally have a sheet-like architecture and round cell morphology, which can create problems in differential diagnosis, particularly in the pediatric population. ${ }^{5}$ When the stromal component shows histological features of malignancy, typically osteosarcoma or chondrosarcoma, we talk of 'myoepithelial carcinoma with heterologous (osteo) sarcomatous differentiation' or 'malignant mixed tumor. ${ }^{5-8}$

Clinically, soft tissue myoepithelial neoplasms appear like longterm painless skin papule or nodule or a polypoid lesion that can be easily confused with other expansive lesions of the soft tissues. Surgery is gold standard treatment: wide excision from lateral margins extended to the deep fascia. Wide excision is mandatory, as myoepithelial carcinoma local recurrence rate is around $40 \%$ and metastatic rate is at least $30 \%$. In children, the disease appears even more aggressive with a rate of recurrence, metastasis and mortality of approximately $50 \%$. Chemotherapy and radiotherapy role are under discussion. We report the case of a patient who is currently followed at our clinic for a head and neck myoepithelial carcinoma of mixed cell type.

\section{Case report}

A 72-year-old Caucasian woman with unremarkable past medical history presented at our clinic for a recently enlarged greyish papula on the scalp. The neoformation developed within few months, it presented as a firm, painless, solid skin tumor of $1 \mathrm{~cm}$ diameter without epidermal ulceration. Clinically differential diagnosis was posed with Merkel-cell carcinoma, sebaceous carcinoma and dermatofibrosarcoma protuberans.

Following surgical removal (complete excision with negative margins, R0), the histological examination disclosed spindle and epithelioid tumor cells with mitotic figures (Figure 2). Immunohistochemical staining showed positive reaction for bcl-2, calponin, cytokeratins (Figure 2B) (Figure 2C), CD99, S100, muscle-
Volume 2 Issue 2 - 2018

\section{Brambullo T,' Kohlscheen E,' Salmaso R, ${ }^{2}$ Cappellesso R, ${ }^{2}$ De Antoni E,' Dalla Venezia E,' Vindigni V,' Bassetto $\mathrm{F}^{\prime}$ \\ 'Department of Plastic Surgery, Padua University, Italy ${ }^{2}$ Department of Pathology, Padua Hospital, Italy}

Correspondence: Erica Dalla Venezia, Department of Plastic Surgery, Padua University, Italy, Email

erica.dallavenezia@gmail.com

Received: June 28, 2017 | Published: March 09, 2018

specific antigen, smooth muscle antigen and vimentin (Figure 2D). Ultrastructurally it was characterized by intermediate filaments, perinuclear tonofilaments and desmosomes (Figure 3): diagnosis of myoepithelial carcinoma of mixed cell type was established on these findings.

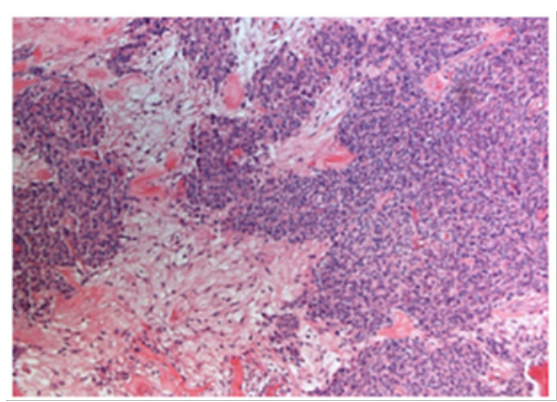

Figure I Double component: sarcomatous component and epithelial component (HE staining I0x).

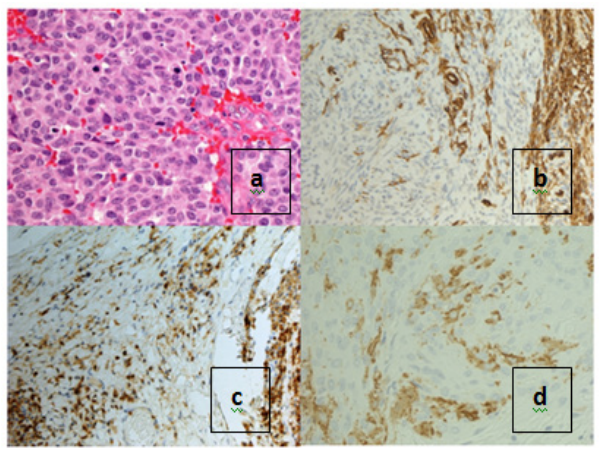

Figure 2 Cutaneous myoephitelial carcinoma: A) Epithelial metastasis $\mathrm{HE}$ staining (40x), B) Cheratine histological staining (10x), C) GFAP histological staining (10x), D) Vimentina histological staining ( I0x).

Local recurrence appeared after one year on the left side of surgical scar, ultrasound detected two pathological laterocervical lymph nodes of the same neck side. A CT scan showed no bone invasion. We performed wide excision of the recurrence and left superficial parotidectomy with selected modified neck dissection type III (Robbin's levels II-IV). 


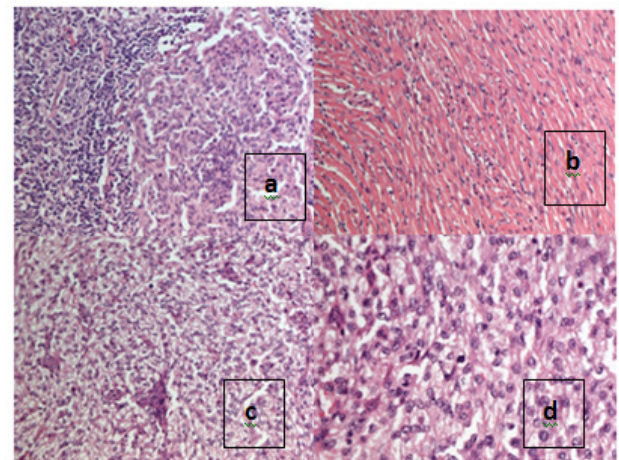

Figure 3 Cutaneous myoepithelial carcinoma: a: Epithelial component, 3b-3d: Sarcomatous component (all HE staining 20x;10x;10x;40x).

Histological examination showed skin and subcutaneous hypodermic recurrence of malignant myoepithelioma and confirmed tumor localization in the pathological lymph nodes (Figure 4); sections of salivary gland and other 23 lymph nodes were free of tumor.

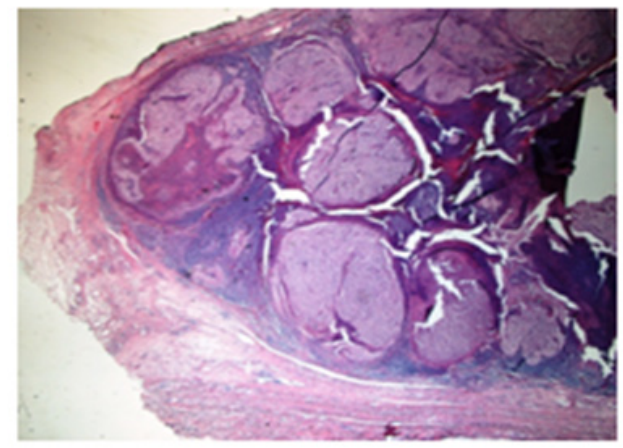

Figure 4 Lymph node metastases of myoephitelial carcinoma (HE staining I,2x).

Reconstruction was performed with split-thickness skin graft (Figure 5). After a three years follow-up of scheduled clinical examination, associated with neck and abdomen ultrasound check, neither local nor lymph node relapse has been documented.

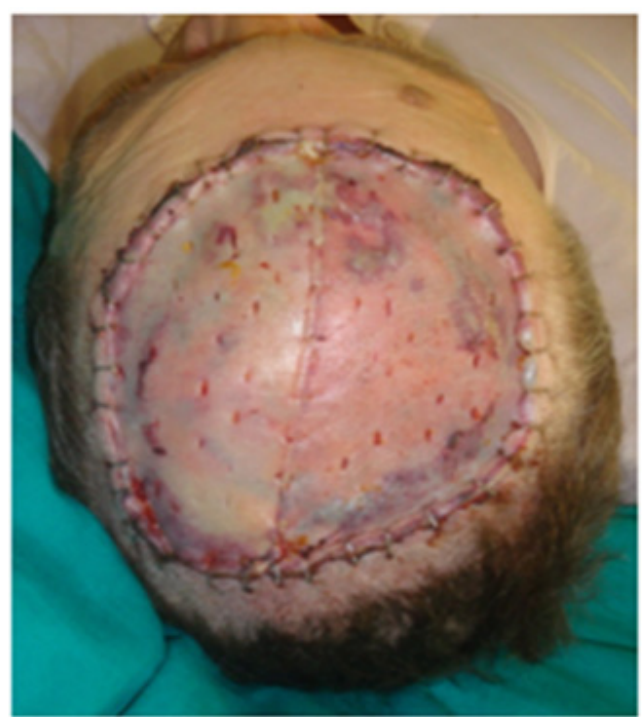

Figure 5 Repair with autologous skin graft.

\section{Discussion}

In 2002, the World Health Organization introduced myoepithelial tumors in the soft tissue neoplasms classification and, in 2003, Hornick et al. ${ }^{5}$ proposed criteria for malignancy. Myoepithelioma and mixed tumor are characterized by benign cytomorphology or mild cytological atypia (low-grade): when moderate atypia is presented (high-grade), the tumor can be classified as myoepithelial carcinoma (epithelioid or spindled cells with vesicular or coarse chromatin, prominent, often large nucleoli, or nuclear pleomorphism) and malignant mixed tumor (cytological malignant cartilage or bone). Hornick et al. ${ }^{5}$ published the most important series of cases and noticed equal sex prevalence and an age peak between thirty and fifty years. Malignant tumors, anyway, were more frequent and more aggressive in children. Head and neck were involved in $15 \%$ of cases, presenting as a nodule as in our case.

The MECa recurrence rate is $36.3 \%$. The most significant univariate factors in the prediction of MECa recurrence are margin status, angiolymphatic invasion, and tumor necrosis and myoepithelial anaplasia. ${ }^{1}$ Regional lymph node metastasis and distant localizations to the lungs, skin, kidney, bone and brain have been reported in $\sim 5 \%$ of cases. The 5 and 10-year disease specific survival rates are 93.5 and $81.8 \%$, respectively. ${ }^{1}$ In our case, we registered a local recurrence after surgery with no involvement of the margins and a lymphatic metastasis. The surgical therapy was sufficient to obtain the complete cure and no chemotherapy or radiotherapy was performed.

\section{Conclusion}

As seen in our clinical case, myoepithelia carcinoma could have an ambiguous clinical behavior because of its histological features. In fact, it consists of clusters of epithelioma like and sarcomatous like cells which make this lesion complex to treat properly. Despite the morphological and histological characterization, more randomized studies are needed to outline the appropriate clinical pathway.

\section{Acknowledgements}

None.

\section{Conflicts of interest}

The author declared that there are no conflicts of interest.

\section{References}

1. Jo VY. Myoepithelial Tumors: An Update. Surg Pathol Clin. 2015;8(3):445-466.

2. Kutzner H, Mentzel T, Kaddu S, et al. Cutaneous myoepithelioma: an under-recognized cutaneous neoplasm composed of myoepithelial cells. Am J Surg Pathol. 2001;25(3):348-355.

3. Lomax-Smith JD, Azzopardi JG. The hyaline cell: a distinctive feature of "mixed" salivary tumours. Histopathology. 1978;2(2):77-92.

4. Jo VY, Fletcher CD. Myoepithelial neoplasms of soft tissue: an update review of the clinicopathologic, immunophenotypic, and genetic features. Head Neck Pathol. 2015;9(1):32-38.

5. Hornick JF, Fletcher CD. Myoepithelial tumors of soft tissue: a clinicopathologic and immunohistochemical study of 101 cases with evaluation of prognostic parameters. Am J Surg Pathol. 2003;27(9):1183-1196. 
6. Kong M, Drill EN, Morris L, et al. Prognostic factors in Myoepithelial Carcinoma of Salivary Glands. A clinicopathologic Study of 48 Cases. Am J Surg Pathol. 2015;39(7):931-938.

7. Thway K, Fisher C. Myoepithelial tumor of soft tissue: histology and genetics of an evolving entity. Adv Anat Pathol. 2014;21(6):411-419.
8. Costigan DC, Doyle LA. Advances in the clinicopathological and molecular classification of cutaneous mesenchymal neoplasms. Histopathology. 2016;68(6):776-795. 